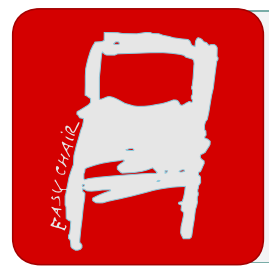

EPiC Series in Health Sciences

Volume 3, 2019, Pages 119-121

CAOS 2019. The 19th Annual Meeting of the International Society for Computer Assisted Orthopaedic Surgery

\title{
Assisted Preoperative Planning in Total Hip Arthroplasty to Reduce Leg Length Discrepancy
}

\author{
Paolo Esposito ${ }^{1}$, Giovanni Francesco Solitro ${ }^{2}$, Farid Amirouche ${ }^{3}$, Damiano \\ Fortuna $^{1}$, Arcangelo Pellegrino ${ }^{1}$, Francesco Villecco ${ }^{1}$ \\ ${ }^{1}$ Department of Industrial Engineering, University of Salerno, Via Giovanni Paolo II 132, \\ Fisciano, 84084, Italy \\ ${ }^{2}$ Department of Orthopaedic Surgery, Louisiana State University Health Science Center of \\ Shreveport, 1501 Kings Hwy, Room 3-317, Shreveport, LA 71104, United States \\ ${ }^{3}$ Department of Orthopaedics, University of Illinois at Chicago, $835 \mathrm{~S}$. Wolcott Ave., Chicago, IL \\ 21287, United States \\ p.esposito23estudenti.unisa.it, gsolit@lsuhsc.edu, \\ Amirouch@uic.edu, daminfor@virgilio.it, apellegrino@unisa.it, \\ fvillecco@unisa.it
}

\section{Introduction}

Preoperative planning is an integral part of total joint arthroplasty, aiming to restore the joint mimicking the native functionality (Choplin 2008). Dimensions of bone and prosthetic implants are usually evaluated by preoperative radiographs to restore leg length discrepancy and femoral offset (Amirouche, Solitro e Walia 2015) . Nevertheless, even when using dedicated software, the femoral component is precisely selected only in $34 \%$ of the cases, resulting in a significant variability in $50 \%$ of the patients with $10 \mathrm{~mm}$ in postoperative leg length discrepancy (Strøm e Reikerås 2018).

With the aim of reducing postoperative complications, in this work a computer algorithm has been developed that uses preoperative $\mathrm{x}$-rays and performs automatic extraction of anatomical landmarks together with an automatic selection of the component that closely restore the native hip.

\section{Methods}

In a first phase, the Fuzzy logic based algorithm extracts the axis of the femoral neck, the femoral axis of the middle diaphysis, both the trochanters and the center of the femoral head. Then, by checking the geometrical data of commercially available hardware (Zimmer Inc., Warsaw, Indiana, U.S.A.) selects the component closer to the native anatomy. In a second phase, by a kinematic approximation 
the algorithm uses the segmentation of the intramedullary canal to position the implant, assuming a press-fit insertion and thus anticipating potential contact region and, consequently, leg length discrepancy and offset.

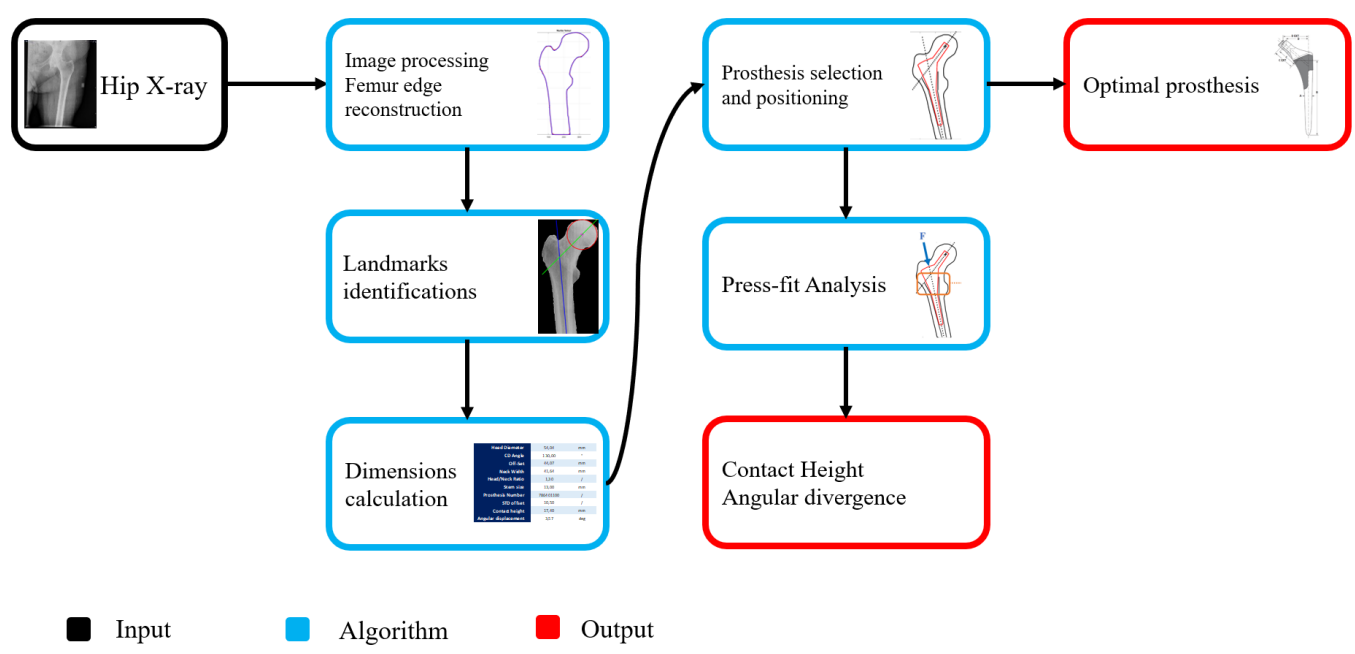

The algorithm has been tested on a set of x-ray images. The following parameters have been extracted: position of femur axis and neck axis, center of rotation, offset, head dimension, neck dimension and angle cervico-diaphysaire (CD angle), dimensions of optimal implant (slightest difference between CD angle of bone and $\mathrm{CD}$ angle of prosthesis), height contact bone-implant and angular divergence of anatomical axis. The correlation diagram is used to determine the femur length bt means of the diameter of the femoral head (Noble 1988).

\section{Results}

A sample of 16 femoral radiographs in Antero-posterior view was used to validate the algorithm. The average femoral head size calculated is $51.32 \mathrm{~mm}$, with a maximum value of $59.27 \mathrm{~mm}$ and a minimum value of $47.85 \mathrm{~mm}$. The head size was used to evaluate the error between the dimension calculated and the one manually measured on the radiograph, resulting in the order of $\pm 2.00 \mathrm{~mm}$. The prediction of the fixation by means of press-fit technique gives an average contact height of $21.49 \mathrm{~mm}$ and an angular deviation of $4.13^{\circ}$ of the neck axis, applying a force of $9.25 \mathrm{kN}$ (Sakai 2011)

\section{Discussion}

Compared to existing methods, the proposed algorithm allows to calculate the femoral sizes necessary for dimensioning the implant from 2D radiographs in the frontal view only and to predict the prosthesisbone contact in terms of height and interference reached after hammering. The algorithm also provides the angular discrepancy generated with the chosen implant, a parameter that indicates the removal from the normal mobility of the limb. The accurate analysis on mobility (ROM) used in other methods (Hsu e Fischer 2018) does not analyze the contact between the femoral stem and the bone, necessary to reach the primary stability of implant. 
It has to be noted that the study, based on coronal x-rays, does not actually account for pelvic tilt (Bono 2018), that will be include in a further research.

\section{REFERENCES}

Amirouche, F., G. Solitro, e A. Walia. (2015). No Effect of Femoral Offset on Bone Implant Micromotion in an Experimental Model. Orthopaedics and Traumatology: Surgery and Research.

Bono, Olivia J. (2018). An Algorithm for Detection and Correction of Pelvic Tilt in Total Hip Replacement. Orthopaedic Surger SURGICAL TECHNOLOGY INTERNATIONAL.

Robert H. Choplin, Christopher N. Henley, Eric M. Edds, William Capello, James L. Rankin, Kenneth A. Buckwalter (2008). Total Hip Arthroplasty in Patients with Bone Deficiency of the Acetabulum. Radiographics : a review publication of the Radiological Society of North America, Inc 28(3), pp 771-786.

Juliana Hsu, Maximilian Fischer, Kunihiko Tokunaga, Gildas Esnault and Klaus Radermacher (2018) Analyzing Bony Constraints as a Key Stone of an Integrated Approach Towards Functional THA Planning.

Noble PC, Alexander JW, Lindahl LJ, Yew DT, Granberry WM, Tullos HS. (1988). The anatomic basis of femoral component design. Clinical Orthopaedics and Related Research.

Sakai R, Takahashi A, Takahira N, Uchiyama K, Yamamoto T, Uchida K, Fukushima K, Moriya M, Takaso M, Itoman M, Mabuchi K. (2011). Hammering force during cementless total hip arthroplasty and risk of microfracture. Hip international: the journal of clinical and experimental research on hip pathology and therapy.

Strøm and Reikerås. (2018). Templating in Uncemented THA. On Accuracy and Postoperative Leg Length Discrepancy. Journal of Orthopaedics. 